\title{
ADOLESCENTES ECUATORIANOS CON ALTOS Y BAJOS NIVELES DE RECHAZO ESCOLAR POR LA BÚSQUEDA DE LA ATENCIÓN DE OTRAS PERSONAS SIGNIFICATIVAS Y SUS DIFERENCIAS CON PERFECCIONISMO
}

\author{
Virginia Narcisa Ortega-Sandoval \\ Universidad Central del Ecuador \\ virortsan@hotmail.com \\ Mạ Pilar Aparicio Flores \\ Universidad de Alicante \\ Aitana Fernández Sogorb \\ Universidad de Alicante \\ José Manuel García Fernández \\ Universidad de Alicante
}

Fecha de Recepción: 13 Mayo 2019

Fecha de Admisión: 25 Septiembre 2019

\section{RESUMEN}

Diversas investigaciones resumen el perfeccionismo como un conjunto de rasgos claramente negativos los cuales son especialmente preocupantes en la etapa de la adolescencia debido a la presión por compañeros y familiares a nivel social, académico, deportivo y cultural entre otros, cuestión que se transforma en un rasgo patológico. Por otro lado, existe un colectivo de población infanto-juvenil que rechaza ir a la escuela con la finalidad de llamar la atención de los demás. Es decir, les crea ansiedad ante la separación de la figura familiar. En el presente estudio se pretendía observar si existen diferencias estadísticamente significativas en perfeccionismo con adolescentes ecuatorianos con elevadas y pequeñas puntuaciones en la búsqueda de la atención de otras personas significativas. Para ello se utilizó el Child-Adolescent Perfectionism Scale (CAPS) y la School Refusal Assessment Scale-Revised for Children (SRAS-R-C). La muestra total de participantes fue de 1920 adolescentes de edades comprendidas entre 15 y 18 años matriculados en cursos entre $1^{0}$ y $3^{\circ}$ de Bachillerato. Las diferencias halladas se dan en ambos grupos de rechazo escolar para el Perfeccionismo Socialmente Prescrito (PSP), Perfeccionismo Auto Orientado-Esfuerzos (PAOE) y el Perfeccionismo Auto Orientado - Crítica (PAO-C), siendo los estudiantes con mayor nivel de rechazo escolar los que puntuaron más elevado en PSP $(d=.17)$, PAO-E $(d=15)$ y PAO-C $(d=.35)$. A este respecto, es necesario ampliar la investigación en este campo de estudio teniendo en cuenta la vulnerabilidad en la personalidad de los adolescentes y la asociación con multitud de trastornos psicopatológicos con los que correlaciona el perfeccionismo según la literatura previa.

Palabras clave: perfeccionismo; rechazo escolar; personas significativas; población infantojuvenil; diferencias. 


\begin{abstract}
Ecuadorian adolescents with high and low levels of school refusal for the search for the attention of other significant people and their differences with perfectionism. Several investigations summarize perfectionism as a set of clearly negative features which is especially worrying in the adolescence stage due to peer and family pressure at social, academic, sports and cultural levels among others, an issue that becomes a feature pathological. On the other hand, there is a group of children and young people who refuse to go to school in order to get the attention of others. That is, it creates anxiety for the separation of the family figure. In the present study, it was intended to observe if there are statistically significant differences in perfectionism with Ecuadorian adolescents with high and small scores in the pursuit of attention from significant others. For this, the Child-Adolescent Perfectionism Scale (CAPS) and the School Refusal Assessment Scale-Revised for Children (SRAS-R-C) were used. The total sample of participants was 1920 adolescents aged between 15 and 18 enrolled in courses between 1st and 3rd Baccalaureate. The differences found are found in both school rejection groups for Socially Prescribed Perfectionism (SPP), Self-Oriented Perfectionism-Strivings (SOP-S) and Self-Oriented Perfectionism-Critical (SOP-C), with the students with the highest level of school rejection those who scored highest in SPP $(d=.17)$, SOP$S(d=.15)$ and SOP-C $(d=.35)$. In this regard, it is necessary to expand research in this field of study taking into account the vulnerability in adolescents' personality and the association with a multitude of psychopathological disorders with which perfectionism correlates according to previous literature.
\end{abstract}

Keywords: perfectionism; school refusal; significant people; infant-youth population; differences

\title{
INTRODUCCIÓN
}

El perfeccionismo es un rasgo de la personalidad complejo debido a la amplia gama de conceptualizaciones desde distintos enfoques tales como el filosófico (Abril, 1984), socioeconómico (Rawls, 2002; Sen, 1998), y psicológico (Adler, 1956; Horney, 1950). Sin embargo, dentro de estas definiciones, una de las más importantes se basa en la tendencia al establecimiento de elevados estándares de desempeño, combinado con una autoevaluación crítica y un incremento en las preocupaciones por cometer errores (Frost, Marten, Lahart y Rosenblate, 1990).

Es por ello, que un gran número de estudios hablan del perfeccionismo como un conjunto de rasgos negativos (Burns, 1980; Haring, Hewitt y Flett, 2003; Hewitt, Flett y Ediger, 1995; Hollender, 1965; Patch, 1984). De hecho, ya en sus estudios iniciales se vinculó con psicopatologías como la neurosis, miedo y dudas al fracaso, y la necesidad de agradar a las personas del entorno más cercano (Adler, 1956; Hamachek, 1978).

A este respecto, resulta especialmente alarmante el constructo en la etapa adolescente, teniendo en cuenta que es una fase de cambios donde los jóvenes desean resaltar ante sus iguales y ocasionalmente sienten presión familiar por obtener niveles más elevados en su ámbito académico, así como social, deportivo y cultural; por lo que en ocasiones se autoimponen un nivel de perfeccionismo insano con rasgos patológicos. Es aquí donde no debemos olvidar el vínculo entre el perfeccionismo y el rechazo escolar, teniendo en cuenta que la negativa a asistir al centro educativo puede cambiar la vida personal de los adolescentes, pero más allá de ello la futura sociedad. De hecho, diversas investigaciones manifiestan que los estudiantes con problemáticas para acudir y permanecer en el aula llevan consigo malas conductas e incluso quejas somáticas, advirtiendo de una prevalencia entre el $5 \%$ y el 28\%, que incluso puede llegar hasta la etapa de bachillerato (Kearney, 2003; Kearney, Lemos y Silverman, 2004. Además de ello, según Kearney (2006), siguiéndole Gonzálvez et al. (2016) en estudios posteriores españoles, cuatro son los factores de los que se compone el rechazo escolar: Evitar la afectividad negativa que provocan estímulos o situaciones relacionadas 
con el ámbito escolar, Escapar de la aversión social o situaciones de evaluación, Búsqueda de la atención de otras personas significativas, y Búsqueda de refuerzos tangibles fuera del ámbito escolar. En este estudio nos centraremos en el tercero de ellos, el cual se enmarca en ese rechazo por llamar la atención de los demás, relacionado con la ansiedad ante la separación de la figura familiar.

Gonzálvez et al. (2015), en un estudio con muestra infanto-juvenil española, afirmaron que variables como el Perfeccionismo Socialmente Prescrito (PSP), el cual se entiende por aquel pensamiento sobre las personas significativas basado en la espera de que uno mismo sea perfecto (Vicent, 2017), predicen el rechazo escolar. Asimismo, Dalton (2017), con población estadounidense, obtuvo resultados similares observando que el perfeccionismo, el cual se marca esos estándares elevados también a nivel de rendimiento académico, manifestaba mayores niveles de rechazo escolar.

A este respecto, bajo nuestro conocimiento no existen investigaciones sobre ambos constructos en población ecuatoriana, lo que nos lleva a marcarnos nuestro objeto de estudio teniendo en cuenta que se trata de una prioridad tanto en el ámbito educativo como familiar. De hecho, teniendo en cuenta la edad y la cultura, nos interesa conocer cuál es la relación entre la búsqueda de la atención de otras personas significativas con las tres variables de perfeccionismo según Vicent (2017) y Ortega-Sandoval (2019) en edad adolescente ecuatoriana. A este respecto, es importante saber, según los autores anteriores que además del PSP, Vicent (2017), en población infantil española halló dos variables de Perfeccionismo Auto Orientado, basados en críticas o esfuerzos; a lo que le siguió Ortega-Sandoval (2019) con población adulta adolescente. En este sentido, el Perfeccionismo Auto Orientado- Esfuerzos (PAO-E), es aquel pensamiento basado en deseos y esfuerzos por conseguir la perfección, y el Perfeccionismo Auto Orientado-Críticas (PA0-C) se compone de un perfeccionismo que marca la autocrítica y el miedo de errar.

\section{OBJETIVOS DE LA INVESTIGACIÓN}

El objetivo de nuestro trabajo es analizar si existen diferencias significativas de las tres dimensiones de perfeccionismo según (Ortega-Sandoval, 2019; Vicent, 2017) en función de elevados y bajos niveles de rechazo escolar debido a la búsqueda de la atención de personas significativas en población adolescente ecuatoriana.

\section{MUESTRA Y/O PARTICIPANTES}

Como muestra la Tabla 1, el total de participantes entrevistados asciende a 1786 alumnos de entre 15 y 18 años de edad (Medad $=16.31 ; D E=1.00$ ), de los cuales un $51 \%$ son de sexo masculino, un $39.6 \%$ se encuentra matriculado en $1^{\circ}$ de Bachillerato, un $33.8 \%$ en $2^{\circ}$ de Bachillerato, y un $26.5 \%$ en 30 de Bachillerato. Asimismo, el $100 \%$ del alumnado participante es matriculado en la ciudad de Quito (Ecuador).

Tabla 1.

Tabla de contingencia sexo* ${ }^{*}$ curso con frecuencias y porcentajes

\begin{tabular}{ccccc}
\hline \multicolumn{5}{c}{ Curso } \\
\hline Sexo & $1^{\circ}$ & $2^{\circ}$ & $3^{\circ}$ & Total \\
Chicos & 366 & 308 & 236 & 910 \\
$\%$ & $20.5 \%$ & $17.2 \%$ & $13.2 \%$ & $51.0 \%$ \\
Chicas & 342 & 296 & 238 & 876 \\
$\%$ & $19.1 \%$ & $16.6 \%$ & $13.3 \%$ & $49.0 \%$ \\
Total & 708 & 604 & 474 & 1786 \\
$\%$ & $39.6 \%$ & $33.8 \%$ & $26.5 \%$ & $100.0 \%$ \\
\hline
\end{tabular}




\section{METODOLOGÍA Y/O INSTRUMENTOS UTILIZADOS}

\section{Instrumentos}

School Refusal Assessment Scale-Revised for Children (SRAS-R-C; Kearney, 2002; 2006): La SRAS-R-C es una escala tipo Likert, con 7 tipos de respuesta $(0=$ Nunca; $6=$ Siempre $)$, que mide el rechazo escolar en niños y jóvenes. El test lo componen 24 ítems divididos en 4 factores: 1). Evitar la afectividad negativa que provocan los estímulos o situaciones relacionadas con el ámbito escolar (e.g.: ¿Cuántas veces te sientes peor al estar en la escuela (por ejemplo, asustado, nervioso o triste) que cuando estás en casa con amigos?); 2). Escapar de la aversión social o situaciones de evaluación (e.g.: ¿Con qué frecuencia evitas ciertos lugares en la escuela (por ejemplo, pasillos, lugares en los que hay grupos de gente) en los que tendrías que hablar con alguien?); 3). Búsqueda de la atención de otras personas significativas (e.g.: ¿Te gustaría estar en casa con tus padres más de lo que les gustaría a los otros chicos(as) de tu edad estar en casa con sus padres?), y 4). Búsqueda de refuerzos tangibles fuera del ámbito escolar (e.g.: Cuando estás en la escuela durante la semana, ¿cuántas veces hablas o te relacionas con otras personas (aparte de tu familia)?). Los niveles de fiabilidad de la versión española fueron aceptables en todos los casos $(\alpha=.70, .79, .87, .72)$ respectivamente (Gonzálvez et al., 2016).

Child and Adolescent Perfectionism Scale (CAPS; Flett, Hewitt, Boucher, Davidson y Munro, 2000; Flett et al., 2016). La versión española de la CAPS (Vicent, 2017), es un instrumento de medida, tipo Likert de 5 puntos ( 1 = Nada cierto en mí; 5 = Muy cierto en mí), compuesta por 13 ítems que se estructuran en 3 dimensiones: 1). Perfeccionismo Socialmente Prescrito (e.g.: La gente espera más de lo que yo soy capaz de dar); 2). Perfeccionismo Auto Orientado-Esfuerzos (e.g.: Siempre intento obtener la puntuación más alta en un examen) y 3). Perfeccionismo Auto OrientadoCríticas (e.g.: No puedo soportar ser menos perfecto). Los niveles de fiabilidad fueron aceptables en todos los casos, tanto para el total de la CAPS $(\alpha=.70)$ como para sus tres dimensiones $(\alpha=.80$, .75 y .72) respectivamente.

\section{PROCEDIMIENTO}

En primer lugar, se llevó a cabo una entrevista entre el equipo investigador y todos los miembros del equipo directivo de las tres instituciones educativas donde se realizó la investigación. Esta entrevista, tuvo como objetivo mantener una primera toma de contacto para poder explicar cuál iba a ser la temática del trabajo de investigación y cuál era la finalidad del mismo. Asimismo, era importante dejar constancia del tipo de cuestionarios, el proceso de administración, el respeto a la privacidad y voluntariedad, y la obtención del consentimiento informado por los progenitores o representantes legales de los entrevistados, teniendo en cuenta que sin este la investigación no se podría realizar. De hecho, un porcentaje de entrevistados fue excluido por no obtener el consentimiento informado por escrito o por omisiones en sus respuestas.

Una vez realizada esta primera toma de contacto, en una segunda sesión, el alumnado fue informado sobre la finalidad del estudio, su voluntariedad y anonimato, y se llevó a cabo la administración de los dos cuestionarios. El tiempo aproximado de la sesión fue alrededor de 20 minutos.

\section{ANÁLISIS DE DATOS}

Para determinar si existen diferencias significativas en PSP, PAO-E Y PAO-C en función del rechazo escolar debido a la búsqueda de la atención de personas significativas, los análisis de datos utilizados fueron pruebas $t$ de Student.

Sus hallazgos fueron interpretados bajo los criterios de Cohen (1988) el cual manifiesta que un pequeño tamaño $=.20-.49$, un tamaño moderado $=.50-.79$, y un gran tamaño $=\leq .80$. 
Del mismo modo, el reconocimiento de jóvenes con elevado o bajo grado del tercer factor de rechazo escolar del SRAS-R-C (Kearney, 2006; Gonzálvez et al., 2016), se consideró mediante las siguientes puntuaciones: un nivel alto con puntuaciones iguales 0 superiores al percentil 75 , y un nivel bajo con puntuaciones iguales 0 inferiores al percentil 25.

Este tipo de análisis fue realizado mediante el programa estadístico SPSS.22.0.

\section{RESULTADOS ALCANZADOS}

La Tabla 2 permite observar que existen diferencias estadísticamente significativas en las variables PSP, PAO-E y PAO-C en función de adolescentes con altas y bajas puntuaciones de rechazo escolar debido a la búsqueda de la atención de otras personas significativas.

Tabla 2.

Diferencias en perfeccionismo en estudiantes con altas y bajas puntuaciones en el FIII del SRAS-R-C

\begin{tabular}{cccccccccccc}
\hline Variable & \multicolumn{2}{c}{$\begin{array}{c}\text { Prueba } \\
\text { Levene }\end{array}$} & \multicolumn{3}{c}{$\begin{array}{c}\text { Altas } \\
\text { puntuaciones }\end{array}$} & \multicolumn{2}{c}{$\begin{array}{c}\text { Bajas } \\
\text { puntuaciones }\end{array}$} & \multicolumn{4}{c}{$\begin{array}{c}\text { Significación estadística y } \\
\text { magnitud diferencias }\end{array}$} \\
\hline & $F$ & $p$ & $M$ & $D E$ & $M$ & $D E$ & $t$ & $g . l$. & $p$ & $d$ \\
\hline PSP & 13.92 & .001 & 17.34 & 4.27 & 16.56 & 4.96 & 2.56 & 776.4 & .011 & .17 \\
PAO-E & 7.91 & .005 & 13.73 & 3.51 & 13.18 & 3.96 & 2.24 & 793.1 & .025 & .15 \\
PAO-C & 3.87 & .049 & 13.07 & 3.58 & 11.77 & 3.84 & 5.40 & 823.2 & $<.001$ & .35 \\
\hline
\end{tabular}

Nota $:$ FIII SRAS = Búsqueda de la atención de otras personas significativas; PSP =

Perfeccionismo Socialmente Prescrito; PAO-E = Perfeccionismo Auto Orientado

Esfuerzos; PAO-C = Perfeccionismo Auto Orientado Crítica

Como se puede observar en las medias que alberga la Figura 1, la magnitud de las diferencias fue baja en todos los casos. Los estudiantes con altos niveles de rechazo escolar debido a la búsqueda de la atención de otras personas significativas obtuvieron puntuaciones más elevadas que sus iguales con bajos niveles para el PSP $(d=17.34)$, PAO-E $(d=13.73)$ y PAO-C $(d=13.07)$. 
Figura 1. Diagrama de barras para las diferencias en puntuaciones de perfeccionismo en estudiantes con altas y bajas puntuaciones en el Factor III del SRAS-R-C.

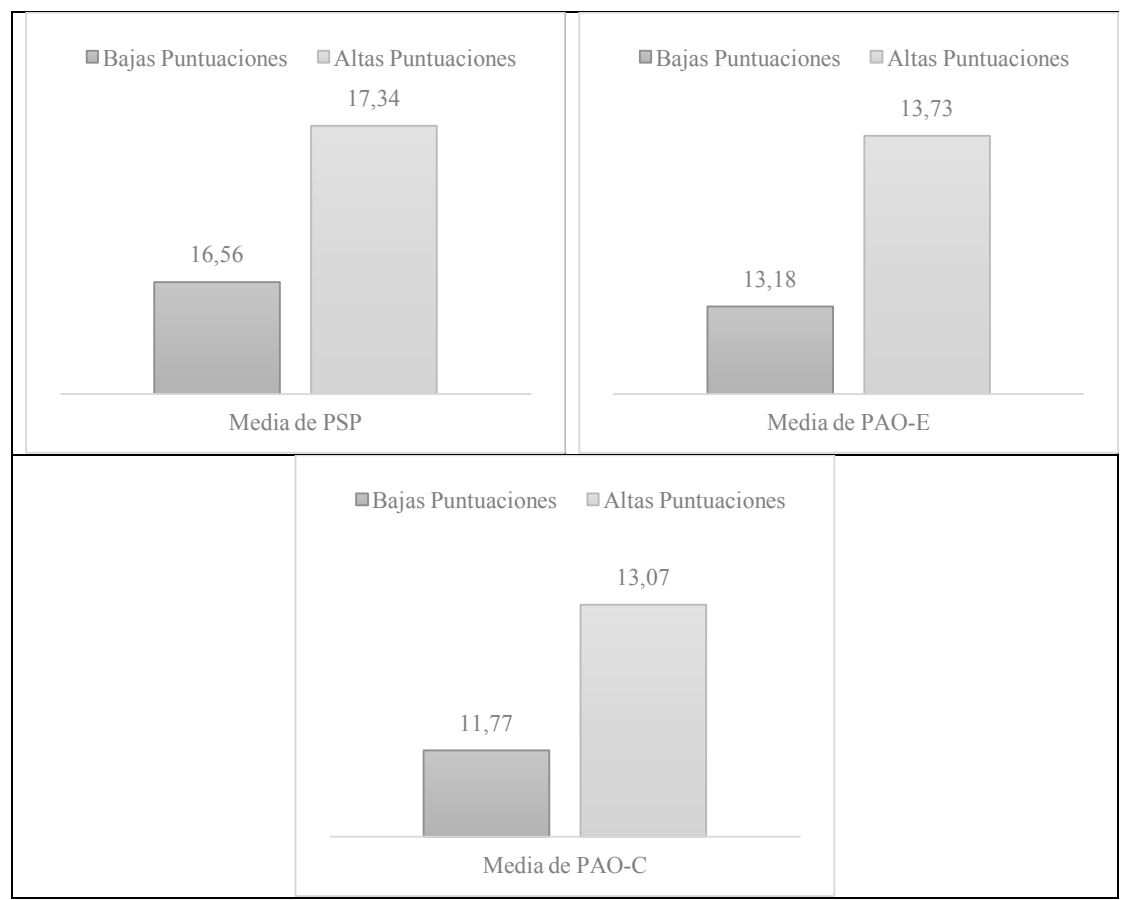

Nota: ${ }^{*}=p<.05 ;{ }^{* *}=p<.01 ;{ }^{* * *}=p<.001$

\section{DISCUSIÓN}

Con el presente trabajo se pretendía analizar si existían diferencias en PSP, PAO-E y PAO-C, dimensiones de la CAPS (Ortega-Sandoval, 2019; Vicent, 2017), en función de las altas y bajas puntuaciones de rechazo escolar según el tercer factor del SRAS-R-C (Kearney, 2006; Gonzálvez et al., 2016), en población adolescente ecuatoriana.

Los hallazgos observados demuestran diferencias estadísticamente significativas, de pequeña magnitud, en PSP, PAO-E y PAO-C.

Cabe destacar que el rechazo escolar por la búsqueda de la atención de otras personas significativas se debe al seguimiento de refuerzos positivos (Gonzálvez et al., 2019). Es decir, este grupo de sujetos suelen ser estudiantes de edades tempranas, los cuales, a partir de llantos y pataletas, manifestado entre otras por ansiedad ante la separación obtienen la atención familiar, permitiendo que permanezcan en el hogar y no en la escuela. Se relaciona, según Sanmartín et al. (2018) con un perfil autodestructivo de afecto, el cual presenta mayores síntomas de ansiedad y depresión.

Gonzálvez et al. (2015), en población infantil española, obtuvieron hallazgos similares a los del presente estudio. Asimismo, los autores justificaron con estudios como el de Essau, Conradt, Sasagawa y Ollendick (2012) y Vicent et al., (2014) que podría deberse a que el perfeccionismo es vinculado positiva y significativamente con la ansiedad y la ansiedad escolar. No obstante, animaron a ampliar la investigación en el factor III del SRAS-R-C con respecto al perfeccionismo, teniendo en cuenta que en este caso la ansiedad generada por la dimensión es ante la separación de un ser que- 
rido. En este sentido, es posible afirmar que el presente estudio, con población adolescente ecuatoriana, obtiene resultados similares a los de Gonzálvez et al. (2015). A este respecto, y teniendo en cuenta que el PSP se trata de un rasgo de la personalidad con propensión a la afectividad negativa (Vicent et al., 2017) debido en parte a la excesiva y rumiante preocupación del constructo (Short y Mazmanian, 2013), es posible que su nivel de preocupación y miedo por cometer errores descienda cuando se sienten protegidos por personas de su entorno.

Del mismo modo, cabe la posibilidad de que las diferencias significativas en PAO-E y PA0-C en función del tercer factor del rechazo escolar sea determinado por la misma razón. A este respecto, cabe destacar, que el estudio realizado por Vicent et al. (2019) demuestra que un grupo con alto PAO-E o PA0-C puntúa significativamente más elevado en afecto negativo y neuroticismo que un grupo con bajos niveles de PAO-C. Esto puede llevar consigo dificultades emocionales en los sujetos que padecen alto PAO-C, y del mismo modo, se sientan seguros, en todo momento, con gente cercana y significativa para ellos.

Este estudio tiene algunas limitaciones que podrían trabajarse en futuras líneas de investigación. En este sentido, no se ha trabajado las diferencias entre sexos. En segundo lugar, no ha sido trabajada las diferencias por cada uno de los cursos escolares. Asimismo, un futuro estudio sería ampliar la muestra a edades inferiores para observar si los hallazgos son semejantes a los del presente estudio.

\section{CONCLUSIONES}

En conclusión, contemplando las futuras consecuencias para la sociedad ecuatoriana, los hallazgos aportados apoyan la importancia de generar estrategias tanto de intervención como de prevención ante casos de perfeccionismo que puedan llevar consigo una influencia de rechazo escolar.

\section{REFERENCIAS BIBLIOGRÁFICAS}

Abril, P. (1984). Aristóteles. Ética a Nicómaco. Barcelona: Orbis

Adler, A. (1956). The neurotic disposition. En H. L. Ansbacher y R. R. Ansbacher (Eds.), The Individual Psychology of Alfred Adler (pp. 239-262). New York: Harper.

Burns, D. D. (1980). The Perfectionist's script for self-defeat. Psychology today. 14(6), 34-52.

Cohen, J. (1988). Statistical power analysis for the behavioral sciences. Hillsdale, NJ: Erlbaum.

Dalton, J. (2017). When Anxiety Affects Learning: How to Help Children with School-Related Anxiety. Center for Anxiety and Behavioral Change. Recuperado de https://www.lcps.org/cms/lib/VA01000195/Centricity/Domain/97/Anxiety.pdf

Essau, C. A., Conradt, J., Sasagawa, S. y Ollendick, T. H. (2012). Prevention of Anxiety Symptoms in Children: Results from a Universal School-Based Trial. Behavior Therapy, 43, 450-464.

Flett, G. L., Hewitt, P. L., Besser, A., Su. C., Vaillancourt, T., Boucher, D., ... Gale, O. (2016). The child-adolescent perfectionism scale: development, psychometric of Psychoeducational Assessment, 34 (7), 634-652. doi: 10.1177/07342829166651381

Flett, G. L., Hewitt, P. L., Boucher, D. J., Davidson, L. A. y Munro, Y. (2000). The Child-Adolescent Perfectionism Scale: development, validation, and association with adjustment. Manuscrito inédito, York University, Toronto, Ontario, Canadá.

Frost, R. O., Marten, P., Lahart, C. y Rosenblate, R. (1990). The dimensions of perfectionism. Cognitive Therapy and Research, 14, 449-468.

Gonzálvez, C., Díaz-Herrero, A., Sanmartín, R., Vicent, M., Pérez-Sánchez, A. M. y García-Fernández, J. M. (2019) Identifying risk profiles of school refusal behavior: differences in social anxiety and family functioning among Spanish adolescents. International Journal of Environmental Research and Public Health, 16 (19), 1-17. doi: 10.3390/ijerph16193731 
Gonzálvez, C., Inglés, C. J., Kearney, C. A., Vicent, M., Sanmartín, R. y García-Fernández, J.M. (2016). School Refusal Assessment Scale-Revised: Factorial Invariance and Latent Means Differences across Gender and Age in Spanish Children. Frontiers in Psychology, 7, 1-10. doi: 10.3389/fpsyg.2016.02011

Gonzálvez, C., Vicent, M., Inglés, C. J., San Martín, N. L., García-Fernández, J. M. y MartínezMonteagudo, M. C. (2015). Diferencias en Perfeccionismo Socialmente Prescrito en función del rechazo escolar. International Journal of Developmental and Educational Psychology, 1 (1), 455461. doi: 10.1760/ijodaep.2015.n1.v1.47

Hamacheck, D. (1978). Psychodynamics of normal and neurotic perfectionism. Psychology. 15, $27-$ 33.

Haring, M., Hewitt, P. L. y Flett, G. L. (2003). Perfectionims, coping, and quality of intimate relationships. Journal of Mariage and Family, 65(1), 143-158. doi: 10.1111/j.17413737.2003.00143.x

Hewitt, P. L., Flett, G. L. y Ediger, E. (1995). Perfectionism traits and perfectionist selfpresentation in eating disorder attitudes, characteristics, and symptoms. International Journal of Eating Disorder, 18, 317-326

Hollender, M. H. (1965). Perfectionism. Comprehensive. Psychiatry, 6 (2), 94-103.

Horney, K. (1950). Neurosis and Human Growth. New York: Norton.

Kearney, C. A. (2003). Brindging the gap among profesionals who addrres youth with school absenteeims: overwiev and suggestions for consensus. Professional Psychology: Reseacrh and practice, 34, 57-65

Kearney, C. A. (2006). Confirmatory factor analysis of the School Refusal Assessment Scale-Revised: Child and parent versions. Journal of Psychopathology and Behavioral Assessment, 28(3), 139-144. doi: 10.1007/s10862-005-9005-6

Kearney, C. A., Lemos, A. y Silverman, J. (2004). The Functional Assessment of School Refusal Behavior. The Behavior Analyst Today, 5 (3), 275-283.

Ortega- Sandoval, V. N. (2019). Rechazo escolar del alumnado de Bachillerato en la ciudad de Quito (Tesis doctoral). Universidad de Alicante, Alicante, España.

Patch, A. R. (1984). Reflections on perfectionism. American Psychologist, 39 (4), 386-390. doi: 10.1037/0003-066x.39.4.386

Rawls, J. (2002). La justicia como equidad. Una reformulación. Barcelona: Paidós

Sanmartín, R., Inglés, C. J., Gonzálvez, C., Vicent, M., Ruiz-Esteban, C. y García-Fernández, J. M. (2018). Impact of affective profiles on school refusal in a Spanish sample of primary education. Journal of Child and Family Studies, 27(4), 1349-1357. doi: 10.1007/s10826-017-0962-7

Short, M. M., y Mazmanian, D. (2013). Perfectionism and negative repetitive thoughts: examining a multiple mediator model in relation to mindfulness. Personality ad Individual Differences, 55, 716-721. doi: 10.1016/j.paid.2013.05.026

Vicent, M. (2017). Estudio del perfeccionismo y su relación con variables psicoeducativas en la infancia tardía (Tesis doctoral). Universidad de Alicante, Alicante, España.

Vicent, M., Inglés, C. J., Sanmartín, R., Gonzálvez, C., Aparicio-Flores, M. P., García-Fernández, J. M. (2019). Clarifying the two facets of Self-Oriented Perfectionism: influences on affect and the Big Five traits of personality in children. Anales de psicología, 35(2), 280-289. doi: 10.6018/analesps.35.2.331441

Vicent, M., Inglés, C. J., Sanmartín, R., Gonzálvez, C., Granados-Alós, L. y García-Fernández, J. M. (2017). Perfeccionismo socialmente prescrito y afectividad en población infantil española. European Journal of Investigation in Health, Psychology and Education, 7 (1), 17-29. doi: 10.1989/ejihpe.v7il.192 


\section{PSICOLOGIA, INFANCIA Y EDUCACIÓN}

Vicent, M., Gonzálvez, C., Lagos-San Martín, N., Gomis, N., Inglés, C. J. y García-Fernández, J. M. (2014). El perfeccionismo como predictor del rechazo escolar. En Espada, Orgilés, Piqueras y Carratalá (comps.), IV Symposium nacional de psicología clínica y de la salud con niños y adolescentes (pp. 73). Madrid: Pirámide. 
